\title{
Iluminación en unidades de cuidados intensivos neonatales: actualización y recomendaciones
}

\author{
Neonatal intensive care unit lighting: update and \\ recommendations
}

\author{
Dr. Roberto G. Rodríguez y Dra. Andrea E. Pattinia
}

\begin{abstract}
RESUMEN
Lograr unailuminación adecuada en las unidades de cuidados intensivos neonatales es un gran desafío: además delas consideraciones habituales de rendimiento visual, costo, energía y estética, aparecen las diferentes necesidades biológicas de los pacientes, profesionales y familiares.

Con el objetivo de revisar los efectos de la luz (natural y artificial) en el marco del cuidado neonatal centrado en el desarrollo, se abordaron los aspectos comunicacionales de la luz, su rol como facilitador de la función visual de los profesionales y sus efectos sobre la fisiología y el desarrollo del neonato. También se revisó el papel de la luz en la regulación del ciclo circadiano del neonato en particular y el uso terapéutico de la luz en general. Para cada aspecto, se especificaron recomendaciones prácticas para lograr un ambiente luminoso adecuado en las unidades de cuidados intensivos neonatales.

Palabras clave: revisión, cuidados intensivos, iluminación, ergonomía.
\end{abstract}

http:/ /dx.doi.org/10.5546/aap.2016.361

\section{INTRODUCCIÓN}

Los constantes avances en neonatología han aumentado la probabilidad de supervivencia de los recién nacidos prematuros y gravemente enfermos. Aunque las unidades de cuidados intensivos neonatales (UCIN) proporcionan atención médica muy especializada, no brindan, necesariamente, el ambiente ideal para el desarrollo del neonato. Pocos ambientes son tan diferentes como el intrauterino y el de una UCIN. ${ }^{1}$ El primero es un econicho que ofrece protección materna, aporte sostenido de nutrientes, temperatura estable y ciclos cronobiológicos, mientras que el último se caracteriza por un patrón de estimulación inapropiado, no contingente, no recíproco y doloroso en el momento de mayor desarrollo estructural y funcional del cerebro. Esta transición a la vida extrauterina impone aún mayores demandas, dadas las limitaciones fisiológicas de los pacientes internados y su capacidad acotada para adaptarse al medio y rechazar estímulos no deseados. El ambiente físico (lumínico, térmico, acústico, radiaciones) de una UCIN es un aspecto crítico, que puede afectar el normal desarrollo del neonato. ${ }^{2} \mathrm{La}$ teoría interactiva del desarrollo indica que el neonato responde activamente al ambiente en busca de un equilibrio, dinámica que ofrece oportunidades de intervenciones preventivas y terapéuticas en su beneficio. ${ }^{3,4}$ Reconocida la individualidad del recién nacido, Sparshott ${ }^{5}$ enumera los aspectos que el ambiente de una UCIN debe proporcionar al neonato: seguridad, confort, postura apropiada, adecuado desarrollo, comunicación, descanso y sueño, muerte digna. Estos principios responden a una aproximación al cuidado médico que conjuga lo tecnológico con lo humano: cuidado centrado en el desarrollo para cubrir las necesidades del recién nacido y su familia.

La Argentina cuenta con iniciativas, manuales y guías para la implementación de este paradigma de atención. ${ }^{6}$ Además, tiene legislación que regula los derechos de padres e hijos durante el proceso de nacimiento (Ley 25929) y una Norma de Organización y Funcionamiento de los Servicios de Neonatología y Cuidados Intensivos Neonatales (Res. 306/2002), que incluye su ambiente físico y, en él, la iluminación. Sin embargo, falta un marco legal/normativo que especifique los parámetros apropiados de iluminación para una UCIN. 
El objetivo de este artículo es revisar los efectos de la iluminación en el neonato y brindar recomendaciones para lograr una iluminación adecuada en el marco del cuidado centrado en el desarrollo. Los aspectos metodológicos de esta revisión pueden consultarse en el anexo electrónico que acompaña esta publicación.

\section{EL AMBIENTE LUMINOSO EN UNA UNIDAD DE CUIDADOS INTENSIVOS NEONATALES}

La iluminación tanto artificial como natural juega varios roles en el ambiente de una UCIN: comunica y transmite sensaciones, soporta la función visual de los profesionales, afecta a la fisiología y el desarrollo del neonato, y regula la función circadiana, además de su uso terapéutico..$^{7-10}$

\section{Aspectos comunicacionales de la luz}

Una UCIN segura y centrada en la familia debe ser pensada como "el primer hogar" del neonato. Debe tener una atmósfera acogedora, que considere el confort de los pacientes y los familiares. ${ }^{11,12}$ La decoración interior y el cuidadoso diseño del ambiente pueden convertir un área clínica de alta tecnología en un espacio más ameno para recién nacidos y familiares. ${ }^{9,13,14}$

La evidencia sugiere que la luz natural (LN) y el contacto con la naturaleza tienen un efecto profundo en la salud y el bienestar humano. ${ }^{15}$ En el ámbito clínico, se han realizado más estudios que abordan posibles efectos positivos de la iluminación en la salud que aquellos relacionados con los efectos positivos de paisajes naturales visibles desde el interior..$^{15}$ Además, la incorporación de sistemas y estrategias de LN ayuda a reducir el consumo eléctrico y mejora la calidad de uso del espacio interior. ${ }^{16-20}$ Para maximizar estos beneficios, el aporte de LN debe planificarse cuidadosamente, ya que existen problemas asociados al ingreso de luz solar sin controlar: dificulta la realización de tareas visuales (deslumbramiento, reflejos en pantallas, manchas de luz), afecta al confort visual y térmico, y contiene radiación indeseada (infrarroja, ultravioleta).

\section{Recomendaciones:}

- Generar puntos de interés, ya sea por contraste o por color, acentuando, por medio de la iluminación, estímulos deseables, como fotografías o imágenes artísticas. ${ }^{20}$

- Lograr un sistema de iluminación flexible, que sea regulable tanto individualmente como por zonas de la Unidad para responder a las dispares necesidades de los neonatos que comparten la UCIN.

- Incorporar LN en el área de internación y ubicar las cunas a más de $60 \mathrm{~cm}$ de las ventanas. El doble vidriado hermético es preferible para minimizar las pérdidas / ganancias de calor.

- Incorporar elementos de control solar externos (parasoles, aleros, celosías) de fácil mantenimiento y limpieza en las ventanas, que permitan un uso flexible según las necesidades. Utilizar colores neutros para minimizar la distorsión del color. ${ }^{21}$

- Evitar radiación solar directa tanto en pacientes como en fluidos intravenosos y pantallas de visualización de datos. Evitar el deslumbramiento en los profesionales. ${ }^{21}$

\section{La luz como soporte de la función visual de los profesionales}

El nivel de iluminación (medido en lux) en las unidades neonatales describió una curva parabólica desde la segunda mitad del siglo XX.22 Las UCIN de la década de los ochenta eran brillantes y luminosas, lo que coincidía con la explosión tecnológica y la mayor sofisticación del cuidado neonatal.

Actualmente, hay una tendencia a utilizar niveles más bajos de iluminación. Dados los sistemas actuales de monitorización, hay menor necesidad de observación directa, por lo que no hace falta luz intensa para controlar a los neonatos. ${ }^{23}$ Existe consenso en que los neonatos están más estables y consumen menos energía en condiciones de baja iluminación, que, además, es necesaria para procedimientos, como ecocardiografías o transiluminación. ${ }^{24}$

Asimismo, en una UCIN, la velocidad, y especialmente la precisión, son muy importantes para el desarrollo de las actividades. Mayores niveles de iluminación permiten una mayor agudeza visual, mejoran la relación señalruido para tareas visuales y la velocidad de las funciones visuales. ${ }^{7}$ Una buena percepción de colores es fundamental para la evaluación clínica de los pacientes. ${ }^{25}$ El índice de rendimiento cromático (IRC) permite evaluar las propiedades de reproducción de color de una fuente de iluminación. Tomando como patrón la LN (IRC $=100)$, este índice mide qué tan "naturales" se perciben los colores al ser iluminados por una fuente de luz artificial. 


\section{Recomendaciones:}

- Un rango de iluminación general de 10 a 600 lux permite evaluar al niño, la coloración de su piel y mucosas, y su perfusión en cualquier lugar de la sala. ${ }^{26}$

- Utilizar fuentes de luz individualizadas de no menos de 2000 lux para la evaluación del neonato o para procedimientos específicos en cortos períodos y evitando exponer a pacientes cercanos. ${ }^{24}$

- Evitar reflejos en las pantallas por medio de iluminación general difusa para no dificultar la lectura de información visual importante, ya sea en las pantallas de monitoreo o durante procedimientos de diagnóstico.

- Las superficies interiores (muros, piso, techo) deben ser claras con terminación mate para que la luz interior se distribuya de manera difusa, para evitar deslumbramiento..$^{9,13}$

- Las fuentes de iluminación artificial deben tener un IRC mayor de $80 .{ }^{21}$ Sus reflectores ópticos deben tener un acabado neutral para mantener las propiedades de reproducción de color.

\section{Efectos de la luz sobre la fisiología y el desarrollo del neonato}

El sistema visual del neonato no está completamente desarrollado al nacer, y se produce, durante el último trimestre, el mayor desarrollo de los sistemas nervioso y visual, que continúan su maduración estructural y funcional durante la infancia. ${ }^{27}$ La iluminación influencia el desarrollo posnatal de la visión y los procesos visuales, así como la maduración de la corteza visual, que es afectada por experiencias visuales prematuras. ${ }^{8,24,28,29}$ Los factores que regulan la cantidad de luz que ingresa al ojo neonatal son biológicos: apertura de los párpados, transmisión a través de ellos, diámetro de la pupila (a partir de las 30-34 semanas de edad posconcepcional) y características de transmisión de los medios oculares. ${ }^{30}$ Hasta alcanzar la edad gestacional correspondiente al término, no es necesaria la luz para el desarrollo visual, y no parece apropiado someter al prematuro a luz intensa cuando intraútero no lo está. ${ }^{31}$

Hay evidencia de que la exposición a luz muy intensa puede producir daño en el ojo inmaduro. ${ }^{32}$ Niveles altos de iluminación han sido asociados a resultados clínicos negativos: menor ganancia de peso, comportamentales y alteraciones en el desarrollo del sueño, además de estrés en pacientes muy prematuros o gravemente enfermos. ${ }^{7,24,33-35} \mathrm{El}$ cambio brusco en la cantidad de luz también afecta al neonato: Shogan y Schumann ${ }^{36}$ reportaron caídas rápidas de saturación en grandes prematuros al aumentar de manera repentina la iluminación. Los neonatos más prematuros son visualmente más vulnerables: se fatigan con facilidad, tienen los párpados muy delgados y su inmadurez les impide cerrar los ojos consistentemente, por lo que son limitados sus recursos de protección frente a la luz. ${ }^{37,38}$ Los cambios en la iluminación ambiental incluyen efectos transitorios: la disminución del nivel de iluminación produce una apertura inmediata y transitoria de los párpados, seguida de un período significativamente más largo cuando se mantiene esta menor iluminación. Los efectos publicados de la reducción de la luz en la UCIN son mayor estabilidad del neonato, estabilidad respiratoria, disminución de frecuencia cardíaca y respiratoria, presión arterial y actividad motora, menor tiempo en asistencia respiratoria y soporte de oxígeno. ${ }^{30}$

\section{Recomendaciones:}

- Limitar la estimulación visual que compita con la información auditiva y táctil predominante en una UCIN evita interferencias sensoriales durante esta etapa de desarrollo.

- Evitar, en todo momento, luz directa en los ojos del neonato.

- Utilizar luces progresivas que permitan un cambio gradual oscuridad-luz para reducir el estrés en el neonato debido al cambio súbito en la iluminación ambiental.

\section{La luz como reguladora del ciclo circadiano del neonato}

Rivkees $^{39}$ advierte que, si bien el feto se desarrolla en oscuridad, se encuentra en un ambiente rico en estimulación sensorial auditiva, táctil y cinestésica. Mantener a los pacientes pretérmino en un ambiente de oscuridad permanente durante su estadía en la UCIN los priva de estímulos circadianos que hubieran recibido durante la gestación. ${ }^{40}$

El reloj biológico fetal es un sistema endógeno que genera ritmos circadianos en respuesta a las señales maternas (actividad, frecuencia cardíaca, cortisol, melatonina, temperatura corporal), al menos, a partir del tercer trimestre de gestación.

Considerando que la luz que alcanza la retina es el principal regulador externo del sistema circadiano humano, en 1997, la Academia Americana de Pediatría recomendó introducir 
ciclos regulares de iluminación diurna/nocturna en las UCIN. ${ }^{41,42}$ Con ello, se busca preservar la vida y brindar el cuidado médico apropiado en un ambiente similar al útero, continuando, en la medida de lo posible, la experiencia que ha sido prematuramente interrumpida.

Las investigaciones apoyan la importancia de los ritmos circadianos para el feto y la relativa falta de estos en el pretérmino. ${ }^{39,40,42-44}$ Hay evidencia de que, entre la semana 28 y la 32 de edad gestacional, la iluminación cíclica tiene consecuencias clínicas positivas en el neonato, pero hay pocos datos del efecto del ritmo circadiano en las funciones fisiológicas, en su crecimiento y en el desarrollo del sistema nervioso central. ${ }^{40,42,45-50}$ Aún no hay consenso sobre cómo introducir en la práctica ciclos circadianos con iluminación artificial ni hay un protocolo de implementación respecto a la duración de ciclos y niveles máximos/mínimos de iluminación. ${ }^{50}$

\section{Recomendaciones:}

- Implementar un esquema de iluminación cíclica. Durante el día, entre 100 y 200 lux, con aportes de luz natural. Por la noche, luz artificial menor de 50 lux, con una distribución espectral semejante a la LN.

- La iluminación diurna/nocturna debe poder llevarse hasta 600 lux con control independiente para luces separadas. ${ }^{51}$

- El uso de cobertores individuales es una alternativa, y se ha comunicado mayor duración media de los períodos de sueño noREM (rapid eye movement; movimiento rápido de ojos) en prematuros estables protegidos por cobertores. ${ }^{52}$

\section{Uso terapéutico de la luz}

La ictericia neonatal es el trastorno más frecuente de la neonatología. Se calcula que el $60 \%-70 \%$ de los recién nacidos lo presentan. ${ }^{53}$ Es un cuadro benigno y autolimitado, que desaparece, generalmente, antes del mes de edad. ${ }^{54}$ Sin embargo, el nivel de bilirrubina no conjugada sérica debe ser monitoreado para evitar niveles tóxicos para el sistema nervioso central. La fototerapia mantiene o baja la concentración de bilirrubina en sangre por medio de la fotodescomposición de sus moléculas. La piel del neonato es expuesta a la luz y, normalmente, se protegen los ojos. Sin embargo, los neonatos en cunas adyacentes no siempre tienen los ojos bloqueados e, incluso, neonatos en tratamiento, a veces, remueven su protección ocular. ${ }^{7}$ Para contrarrestar esto, las mantas de fibra óptica que envuelven al neonato son una alternativa ya publicada. ${ }^{55}$

Con un pigmento macular menos denso y un medio ocular que transmite más energía de longitud de onda corta (azules) y ultravioleta cercano, el neonato tiene mayor riesgo de sufrir daño ocular por luz de onda corta, entre otras muchas susceptibilidades asociadas al sistema visual en desarrollo. ${ }^{56}$ Con un pico de sensibilidad en los $440 \mathrm{~nm}$, la exposición a esta región del espectro electromagnético podría producir daño oxidativo en los fotorreceptores e impedir su normal proceso de regeneración. Este proceso fue descrito en estudios in vitro y en modelos animales. ${ }^{57-59}$ Tanto el sistema circadiano humano como la fotodescomposición de la bilirrubina requieren de luz de onda corta, procesos que tienen su mayor sensibilidad espectral en los 450 $\mathrm{nm}$, región adyacente al pico de $440 \mathrm{~nm}$ de la función de daño por luz azul. ${ }^{8,60}$

La retinopatía del prematuro (retinopathy of prematurity; ROP, por sus siglas en inglés) es una causa importante de ceguera. Presentan mayor riesgo los nacidos con menos de $1500 \mathrm{~g}$ y/o menos de 32 semanas de edad gestacional. Este grupo debe ser controlado por exámenes oftalmológicos seriados hasta verificar que se haya completado la maduración retinal. Cuando la enfermedad se describió por primera vez, se sugirió que la exposición temprana de la retina a la luz podría aumentar el número de radicales libres, lo que contribuye al desarrollo de ROP. ${ }^{61}$ Desde la década de los cincuenta del siglo XX, se sabe que el uso excesivo de oxígeno aumenta el riesgo de ROP. ${ }^{62}$ Estudios en animales demostraron lesiones de la retina luego de una exposición a luces extremadamente brillantes durante períodos prolongados, algo no practicado, por lo general, en una UCIN. 10,33,63,64 El interés por la luz fue reavivado tras el trabajo de Glass y colaboradores, ${ }^{65}$ que reportaron una reducción en la incidencia de $\mathrm{ROP}$ al disminuir la iluminación en la UCIN. En busca de dar una respuesta definitiva acerca del efecto de la luz en la ROP, se condujo un estudio multicéntrico aleatorizado denominado LIGHT-ROP, que no encontró diferencias significativas en la gravedad de ROP entre neonatos expuestos y no expuestos a la luz. ${ }^{66}$ Si bien, generalmente, se acepta que la luz no es un factor de riesgo para el desarrollo de ROP, sigue siendo un tema controversial. ${ }^{67-69}$ Según Fielder y Moseley, ${ }^{30}$ la luz no afecta al desarrollo de ROP, pero su rol en la patogénesis 
de otras secuelas oftalmológicas del nacimiento pretérmino es desconocido.

\section{Recomendaciones:}

- Las lámparas en la región azul del espectro (460-490 nm) son las más efectivas en el tratamiento de la hiperbilirrubinemia.

- Al utilizar lámparas con fines terapéuticos, limitar la intensidad de la fuente de iluminación al mínimo requerido por la tarea y reducir al mínimo el tiempo de exposición.

- Evitar la exposición de radiación ultravioleta e infrarroja en los ojos del neonato a través del uso de lámparas apropiadas, lentes o filtros.

\section{CONCLUSIONES}

Lograr una iluminación adecuada en una UCIN es un gran desafío. Además de consideraciones relativas al rendimiento visual, costo, energía y estética, deben atenderse las necesidades biológicas de los pacientes, profesionales de la salud y familiares, muy diferentes entre sí.

Un creciente cuerpo de conocimiento se ha desarrollado en torno a los efectos positivos y/o negativos de los factores ambientales en el neonato, agrupados en un campo disciplinar relativamente reciente: la neonatología ambiental. Sin embargo, es menor la disponibilidad de guías y recomendaciones que permitan la aplicación práctica de dicho conocimiento científico.

Esta actualización se valió de diversas fuentes (legales, empíricas, técnicas, científicas) para presentar el estado actual respecto al medio ambiente visual de una UCIN. Lejos de ser exhaustiva, busca servir de guía para definir racionalmente la iluminación del área de internación sin descuidar las complejas necesidades de los pacientes, familiares y profesionales.

Por último, este documento busca ser un disparador para generar, a través de la discusión y el consenso, un marco de referencia legal y normativo propio de nuestro país, según los preceptos del cuidado neonatal centrado en el desarrollo. Lograr un ambiente luminoso seguro y que favorezca el desarrollo del neonato requiere de intervenciones de baja tecnología y poca inversión económica (en relación con el equipamiento de una UCIN), pero de alto rendimiento considerando los beneficios en el neonato aquí descritos.

\section{REFERENCIAS}

1. Pallas Alonso CR, Gutiérrez Argul O. El ambiente en los cuidados intensivos neonatales. An Esp Pediatr 1997;47(6):618-20.

2. Hunt KN. The NICU: Environmental Effects of the Neonatal Intensive Care Unit on Infants and Caregivers. Research Papers 2011. Paper 71. [Acceso: 12 de enero de 2016]. Disponible en: http://opensiuc.lib.siu.edu/cgi/ viewcontent.cgi? article $=1068 \&$ context $=$ gs_rp.

3. Als H. A synactive model of neonatal behavioral organization: framework for the assessment of neurobehavioral development in the premature infant and for support of infants and parents in the neonatal intensive care environment. Phys Occup Ther Pediatr 1986;6(3-4):3-53.

4. Franck LS, Lawhon G. Environmental and behavioral strategies to prevent and manage neonatal pain. Semin Perinatol 1998;22(5):434-43.

5. Sparshott M. Pain, distress and the newborn baby. UK: Wiley-Blackwell; 1996.

6. Larguía AM, González MA, Solana C, Basualdo MN, et al. Maternidad Segura y Centrada en la Familia. (MSCF): Conceptualización e implementación del modelo. Buenos Aires: UNICEF, 2011. [Acceso: 1 de marzo de 2016]. Disponible en: http://www.unicef.org/argentina/ spanish/GUIA_MSCF.pdf.

7. Bullough J, Rea MS. Lighting for neonatal intensive care units: some critical information for design. Light Res Technol 1996;28(4):189-98.

8. Rea M. Lighting for caregivers in the neonatal intensive care unit. Clin Prinatol 2004;31(2):229-42.

9. Rizzo P, Rea M, White R. Lighting for today's neonatal intensive care unit. Newborn Infant Nurs Rev 2010;10(2): 107-13.

10. Lai TT, Bearer CF. Iatrogenic environmental hazards in the neonatal intensive care unit. Clin Perinatol 2008;35(1): 163-81.

11. Cooper LG, Gooding JS, Gallagher J, Sternesky L, et al. Impact of a family-centered care initiative on NICU care, staff and families. J Perinatol 2007;27(Suppl 2):S32-7.

12. Laing I, Ducker T, Leaf A, Newmarch P. Designing a NeonatalUnit. Report for the British Association of Perinatal Medicine, 2004. [Acceso: 1 de marzo de 2016]. Disponible en: http://www.bapm.org/publications/documents / guidelines/DesigningNNU_May2004b.pdf.

13. White RD. Lighting in the neonatal intensive care unit: practical applications of scientific principles. Clin Perinatol 2004;31(2):323-30.

14. Dalke H, Little J, Niemann E, Camgoz N, et al. Colour and lighting in hospital design. Opt Laser Technol 2006;38(46):343-65.

15. BeuteF, de KortYA. Salutogenic effects of the environment: Review of health protective effects of nature and daylight. Appl Psychol Health Well Being 2014;6(1):67-95.

16. Boyce P, Hunter C, Howlett O. The benefits of Daylight through Windows. Troy, New York: Rensselaer Polytechnic Institute, 2003. [Acceso: 1 de marzo de 2016]. Disponible en: http:/ / thedaylightsite.com/wp-content/uploads / papers/DaylightBenefits.pdf.

17. Leslie R. Capturing the daylight dividend in buildings: why and how? Buil Environ 2003;38(2):381-5.

18. Joseph A. The Impact of Light on Outcomes in Healthcare Settings. Issue Paper 2, 2006. Concord, CA: The Center for Health Design. [Acceso: 12 enero 2016]. Diponible en: http:/ /www.healthdesign.org/sites/default/files/CHD_ Issue_Paper2.pdf.

19. Alzoubi H, Al-Rqaibat S, Bataineh RF. Pre-versus postoccupancy evaluation of daylight quality in hospitals. Buil Environ 2010;45(12):2652-65. 
20. Iyendo TO, Alibaba HZ. Enhancing the Hospital Healing Environment through Art and Day-lighting for User's Therapeutic Process. IJAC 2014;3(9):101-19.

21. WhiteRD,SmithJA,ShepleyMM. Recommended standards for newborn ICU design, eighth edition. J Perinatol 2013;33(Suppl 1):S2-16.

22. García Sánchez P. Cuidado neonatal con atención al desarrollo. Rev Esp Ped 2002;58(343):28-36.

23. Young J. The visual system. In Young J, ed. Developmental Care of the Premature Baby. London: Baillieré Tindall; 1996. Págs.21-33.

24. White RD. Recommended standards for the newborn ICU. J Perinatol 2007;27(Suppl 2):S4-19.

25. Figueiro MG, Rea MS, Boyce P, White R, Kolberg K. The effects of bright light on day and night shift nurses' performance and well-being in the NICU. Neonatal Intensive Care 2001;14(1):29-32.

26. DiLaura D, Houser KW, Mistrick RG, Steffy GR (eds). The Lighting Handbook 10th ed: Reference and Application. New York: Illuminating Engineering Society of North America; 2011.Pág.120.

27. Birch EE, O'Connor AR. Preterm birth and visual development. Semin Neonatol 2001;6(6):487-97.

28. Logvinov SV, Potapov AV, Varakuta EY, Drobatulina DA. Dynamics of structural changes in the retina during long-term exposure to bright light. Bull Exp Biol Med 2003;136(4):411-4.

29. Szczepa skiM,Kamianowska M. Theillumination intensity in the neonatal intensive care unit. Arch Perinat Med 2008;14(2):47-50.

30. Fielder AR, Moseley MJ. Environmental Light and the Preterm Infant. Semin Perinatol 2000;24(4):291-8.

31. UK Department of Health. Guidance for the design of hospital neonatal units including special care, family spaces and specialist clinical rooms. Health Building Note 03.09: Neonatal units. 2013. [Acceso: 1 de marzo de 2016]. Disponible en: https:/ / www.gov.uk/government/ uploads/system/uploads/attachment_data/file/147879/ HBN_09-03_Final.pdf.

32. Havelius U, Hansen F, Hindfelt B, Krakau T. Human ocular vasodynamic changes in light and darkness. Invest Ophthalmol Vis Sci 1999;40(8):1850-5.

33. Pickler RH,McGrathJM, Reyna BA, Tubbs-Cooley HL, etal. Effects of the neonatal intensive care unit environment on preterm infant oral feeding. Res Rep Neonatol 2013;2013(3): $15-20$.

34. SheldonSH, BellE. Light, Sleep, and Development. Pediatrics 1987;79(6):1053-4.

35. Peng N,Bachman H, Jenkins R, Chen $\mathrm{CH}$, etal. Relationships between environmental stressors and stress biobehavioral responses of preterm infants in NICU. J Perinat Neonatal Nurs 2009;23(4):363-71.

36. Shogan MG, Schumann LL. The effect of environmental lighting on the oxygen saturation of preterm infants in the NICU. Neonatal Netw 1993;12(5):7-13.

37. Robinson J, Fielder AR. Pupillary diameter and reaction to light in preterm neonates. Arch Dis Child 1990;65(1 Spec No):35-8.

38. Robinson J, Fielder AR. Light and the immature visual system. Eye (Lond) 1992;6(Pt 2):166-72.

39. Rivkees SA. Developing circadian rhythmicity in infants. Pediatrics 2003;112(2):373-81.

40. Rivkees SA. Emergence and influences of circadian rhythmicity in infants. Clin Perinatol 2004;31(2):217-28.

41. Moore-Ede MC, Sulzman FM, Fuller CA. The Clocks That Time Us: Physiology of the Circadian Timing System. Cambridge, MA: Harvard University Press; 1982.

42. Mirmiran M, Ariagno RL. Influence of light in the NICU on the development of circadian rhythms in preterm infants. Semin Perinatol 2000;24(4):247-57.

43. McGraw K, Hoffmann R, Harker C, Herman JH. The development of circadian rhythms in a human infant. Sleep 1999;22(3):303-10.

44. Figueiro MG, Appleman K, Bullough JD, Rea MS. A discussion of recommended standards for lighting in the newborn intensive care unit. J Perinatol 2006;26:S19-26.

45. Mann NP, Haddow R, Stokes L, Goodley S, Rutter N. Effect of night and day on preterm infants in a newborn nursery: randomised trial. Br Med J (Clin Res Ed) 1986;293(6557): 1265-7.

46. MillerCL, White R, Whitman TL, O'Callaghan MF, Maxwell SE. The effects of cycled verses noncycled lighting on growth and development in preterm infants. Infant Behav Dev 1995;18(1):87-95.

47. Brandon DH,Holditch-Davis D, Belyea M. Preterm infants born at less than 31 weeks' gestation have improved growth in cycled light compared with continuous near darkness. J Pediatr 2002;140(2):192-9.

48. Jung IS. Effects of cycled lighting on body weight, physiological variables and behavioral states in low birth weight infants. Taehan Kanho Hakhoe Chi 2005;35(1):143-53.

49. Begum E, Bonno M, Obata M, Yamamoto H, etal. Emergence of physiological rhythmicity in term and preterm neonates in a neonatal intensive care unit. J Circadian Rhythms 2006;4(1):11.

50. Morag I, Ohlsson A. Cycled light in the intensive care unit for preterm and low birth weight infants. Cochrane Database Syst Rev 2011;(1):CD006982.

51. García del Río M, Sánchez Luna M, Doménech Martínez E, Izquierdo Macián I, et al. Revisión de los estándares y recomendaciones para el diseño de una unidad de neonatología. An Pediat (Barc) 2007;67(6):594-602.

52. Hellstrom-Westas L, Inghammar M, Isaksson $\mathrm{K}$, Rosen I, Stjernqvist K. Short-term effects of incubators covers on quiet sleep in stable premature infants. Acta Paediatr 2001;90(9):1004-8.

53. Ríos GM. Síndrome ictérico del primer trimestre. Rev Chil Pediatr 2002;73(4):399-401.

54. Martínez JC. El real problema del recién nacido ictérico. Nuevas guías de la Academia Estadounidense de Pediatría. Arch Argent Pediatr 2005;103(6):524-32

55. Rea MS, ed. IESNA Lighting Handbook. 9th ed. New York: Illuminating Engineering Society of North America; 2000.

56. Fulton A, Abramov I, Allen J, Gwiazda J, et al. Optical radiation effects on visual development. En Waxier $\mathrm{M}$, Hitchins VM, eds. Optical Radiation and Visual Health. Boca Raton, Fla: CRC Press, Inc.; 1986:137-46.

57. Ham WT, Mueller HA, Sliney DH. Retinal sensitivity to damage from short wavelength light. Nature 1976;260(5547):153-5.

58. Van Norren D, Schellekens P. Blue light hazard in rat. Vision Res 1990;30(10):1517-20.

59. Sperling HG. Prolonged intense spectral light effects on rhesus retina. In Williams TP, Baker BN, eds. The Effects of Constant Light on Visual Processes. Nueva York: Plenum Press; 1980.Págs.195-214.

60. Gies HP, Roy CR. Bilirubin phototherapy and potential UVR hazards. Health Phys 1990;58(3):313-20.

61. Terry TL. Extreme prematurity and fibroblastic overgrowth of persistent vascular sheath behind each crystalline lens. Am J Ophthalmol 1942;25(2):203-4.

62. Campbell K. Intensive oxygen therapy as a possible cause of retrolental fibroplasias: a clinical approach. Med J Aust 1951;2(2):48-50.

63. Kuwabara T, Funahashi M. Lightdamage in the developing rat retina. Arch Ophthalmol 1976;94(8):1369-74. 
64. Messner KH, Maisels MJ, Leure-DuPree AE. Phototoxicity to the newborn primate retina. Invest Ophthalmol Vis Sci 1978;17(2):178-82.

65. Glass P, Avery GB, Subramanian KNS, Keys MP, et al. Effect of bright light in the hospital nursery on the incidence of retinopathy of prematurity. N Engl J Med 1985;313(7): 401-4.

66. Reynolds JD, Hardy RJ, Kennedy KA, Spencer R, et al Lack of efficacy of light reduction in preventing retinopathy of prematurity. Light reduction in retinopathy of prematury (LIGHT-ROP) Cooperative Group. N Engl J Med 1998;338(22):1572-6.

67. Landry RJ, Scheidt PC, Hammond RW. Ambient Ligth and Photothepary Conditions of Eigth Neonatal Care Units: a sumary report. Pediatrics 1985;75(2 Pt 2):434-6.

68. Phelps DL, Watts JL. Early light reduction for preventing retinopathy of prematurity in very low birth weight infants. Cochrane Database Syst Rev 2001;1:D000122.

69. Antonucci R, Porcella A, Fanos V. The infant incubator in the neonatal intensive care unit: unresolved issues and future developments. J Perinat Med 2009;37(6):587-98.

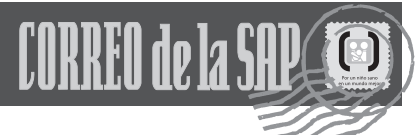

Los siguientes resúmenes y comentarios de trabajos seleccionados se encuentran disponibles en la versión electrónica de este número.

\section{PEDIATR PULMONOL 2015;50:1294-300}

Transferencia de los pacientes dependientes de tecnología respiratoria desde el cuidado pediátrico al de adultos (Agarwal A, et al. Transition of Respiratory Technology Dependent Patients from Pediatric to Adult Pulmonology Care)

Comentario: Dra. Verónica Aguerre. Servicio de Neumonología. Hospital de Pediatría "Prof. Dr. Juan P. Garrahan".

N ENGL J MED 2016;374(9):833-42

Reducción en la mortalidad alejada entre los supervivientes a 5 años de cáncer infantil (Armstrong GT, et al. Reduction in Late Mortality among 5-Year Survivors of Childhood Cancer)

Comentario: Dra. Marcela María Palladino. Cuidados intermedios y moderados. Hospital de Pediatría "Prof. Dr. Juan P. Garrahan".

N ENGL J MED 2016;374(12):1111-22

Alimentación parenteral temprana o tardía en niños críticamente enfermos (Fivez $T$, et al. Early versus Late Parenteral Nutrition in Critically Ill Children)

Comentario: Dr. Pablo Eulmesekian. Servicio Terapia Intensiva Pediátrica. Hospital Italiano de Buenos Aires.

PEDIATRICS 2016;137(3):1-12

Riesgo de cáncer en niños concebidos con tecnologías de reproducción asistida (Reigstad MM, et al. Risk of Cancer in Children Conceived by Assisted Reproductive Technology)

Comentario: Dra. Ma. Florencia Tisi Baña. Servicio de Hematología Oncología Pediátrica y Trasplante de Médula Ósea. Hospital Italiano de Buenos Aires.

CHEST 2016;149(1):120-42

Niños con tos crónica húmeda o productiva - Estudios y tratamiento: Una revisión sistemática

(Chang AB, et al. Productive Cough-Treatment and Investigations: A Systematic Review)

Comentario: Dr. Fernando Rentería. Sala de Neumonología. Hospital Sor María Ludovica. La Plata.

JAMA PEDIATR 2016;170(2):163-70

Efectos endocrinos de los corticoides inhalados en niños.(Kapadia $C R$, et al. Endocrine Effects of Inhaled Corticosteroids in Children)

Comentario: Dr. Conrado Llapur. Hospital del Niño Jesús. Tucumán.

N ENGL J MED 2016;374(18):1733-43

Ensayo aleatorizado sobre la introducción de alimentos alergénicos en niños alimentados con lactancia materna (Perkin MR, et al. Randomized Trial of Introduction of Allergenic Foods in Breast-Fed Infants) Comentario: Dr. Ricardo J. Saranz. Servicio de Alergia e Inmunología, Clínica Universitaria Reina Fabiola. Córdoba. 


\section{ANEXO}

\section{Metodología}

Se realizó una búsqueda de antecedentes, que abarcó las leyes (obligatorias) y normas (voluntarias) de vigencia en Argentina, normas y estándares internacionales, guías de recomendaciones de asociaciones profesionales, tesis de posgrado, informes técnicos y académicos, junto con artículos científicos con referato. El motor de búsqueda fue Google Académico (Scholar Google), y se utilizaron las siguientes palabras clave y sus combinaciones (en inglés): NICU, hospital, healthcare, lighting, natural light, developmental care, environmental factors, human factors, environmental neonatology, standards, guidelines, checklist, design, preterm, jaundice, blue light hazard. Y, en español: UCIN, hospital, iluminación, luz natural, cuidado centrado en el desarrollo, maternidad centrada en la familia, neonatología ambiental, estándares, guías, lista de chequeo, diseño, prematuros, ictericia neonatal. Se acotó la búsqueda a publicaciones de entre 1990 y 2015. A partir de una búsqueda inicial y de la lectura crítica de los documentos, se detectaron autores y publicaciones clave, y se amplió el corpus documental con algunas publicaciones anteriores al período seleccionado sobre la base de su relevancia en términos de originalidad e impacto. Como resultado, se seleccionaron 70 documentos técnicos y científicos, que constituyeron la fuente de este artículo de actualización. 